\title{
Knockdown of RPL9 expression inhibits colorectal carcinoma growth via the inactivation of Id-1/NF-kB signaling axis
}

\author{
IN HYE BAIK ${ }^{1,2^{*}}$, GUK-HEUI JO ${ }^{3 *}$, DAEKWAN SEO ${ }^{4}$, MIN JI KO ${ }^{1}$, \\ CHI HEUM CHO ${ }^{5}$, MIN GOO LEE ${ }^{2}$ and YUN-HAN LEE ${ }^{1}$ \\ ${ }^{1}$ Department of Molecular Medicine, Keimyung University School of Medicine, Daegu; \\ ${ }^{2}$ Department of Pharmacology and Brain Korea 21 Project for Medical Sciences, Yonsei University College of Medicine, \\ Seoul; ${ }^{3}$ Myunggok Eye Research Institute, Kim's Eye Hospital, Konyang University College of Medicine, Seoul; \\ ${ }^{4}$ Severance Biomedical Science Institute, Brain Korea 21 PLUS Project for Medical Science, \\ Yonsei University College of Medicine, Seoul; ${ }^{5}$ Department of Obstetrics and Gynecology, \\ Keimyung University School of Medicine, Daegu, Republic of Korea
}

Received June 30, 2016; Accepted August 25, 2016

DOI: $10.3892 / \mathrm{ijo} .2016 .3688$

\begin{abstract}
Ribosomal protein L9 (RPL9), a component of the 60S subunit for protein synthesis, is upregulated in human colorectal cancer. In the present study, we investigated whether RPL9 gained extraribosomal function during tumorigenesis and whether targeting of RPL9 with small interfering (si) RNA could alter the course of colorectal cancer progression. Our results showed that siRNA knockdown of RPL9 suppresses colorectal cancer (CRC) cell growth and long-term colony formation through an increase in sub-G1 cell population and a strong induction of apoptotic cell death. To obtain insights into the molecular changes in response to RPL9 knockdown, global changes in gene expression were examined using RNA sequencing. It revealed that RPL9-specific knockdown led to dysregulation of 918 genes in HCT116 and 3178 genes in HT29 cells. Among these, 296 genes showed same directional regulation (128 upregulated and 168 downregulated genes) and were considered as a common RPL9 knockdown signature. Particularly, we found through a network analysis that Id-1, which is functionally associated with activation of NF- $\mathrm{B}$ and cell survival, was commonly downregulated. Subsequent western blot analysis affirmed that RPL9 silencing induced the decrease in the levels of Id- 1 and phosphorylated I $\kappa \mathrm{B} \alpha$ in both HCT116 and HT29 cells. Also, the same condition decreased
\end{abstract}

Correspondence to: Professor Yun-Han Lee, Department of Molecular Medicine, Keimyung University School of Medicine, 1095 Dalgubeol-daero, Dalseo-gu, Daegu 42601, Republic of Korea E-mail: yhlee87@kmu.ac.kr

*Contributed equally

Key words: colorectal cancer, RPL9, RNA interference, extraribosomal function, Id-1, NF- $\mathrm{KB}$, apoptosis the levels of PARP-1 and pro-caspase-3, accelerating apoptosis. Furthermore, inhibition of RPL9 expression significantly suppressed the growth of human CRC xenografts in nude mice. These findings indicate that the function of RPL9 is correlated with Id-1/NF- $\kappa \mathrm{B}$ signaling axis and suggest that targeting RPL9 could be an attractive option for molecular therapy of colorectal cancer.

\section{Introduction}

Colorectal cancer is the third most common cancer worldwide and the fourth leading cause of cancer-related death (1). The patients diagnosed with colorectal cancer often develop colorectal metastases and approximately $80-90 \%$ of these patients are found with unresectable metastatic liver disease (2). Colorectal cancer is associated with the progressive accumulation of mutations in oncogenes and tumor suppressor genes. These tumor-specific mutations are the key to understand the cellular processes underlying tumorigenesis and could be used as diagnostic and therapeutic targets (3).

Ribosomal proteins (RPs) are one of the components of ribosomes and there are approximately 80 RPs in eukaryotes (4). RPs are known to stabilize specific rRNA structures in mature ribosomal subunits and promote correct folding of rRNAs during ribosomal assembly. However, not only RPs have crucial role in protein biosynthesis, but recent studies have also associated them to human congenital disorders and cancers (5). For example, RPS4 has been implicated in Turner's syndrome (6), and mutant RPS19 was found in individuals with Diamond-Blackfan anemia (7). Hence, the functions of RPs that are independent of protein biosynthesis are called extraribosomal functions. The extraribosomal functions include transcription and repair, RNA splicing and modification, cell growth and proliferation, regulation of apoptosis and development (8). Moreover, knockdown of many individual RPs resulted in p53 accumulation, cell death and defective development $(8,9)$. Various RPs have been found to be overexpressed in many cancer cells and are associated with 
the development and progression of malignant cancers (9). For instance, in gastric cancer cell line, RPL6 promoted cell cycle progression and cancer cell proliferation by upregulating the expression of cyclin E (10). Also, phosphorylated RPS3 activated anti-apoptotic protein TRAF2 and enhanced radioresistance in non-small cell lung cancer cells (11).

Ribosomal protein L9 (RPL9) is a component of the 60S subunit that belongs to the L6P family of ribosomal proteins. From what we have gathered, RPL9's extraribosomal function is not known, but one of the studies have suggested that human RPL9 appears to be involved with the uncontrolled growth by promoting stress-mediated survival and that RPL9 is more like anti-apoptotic-encoding RP genes in yeast (12). In addition, there are reports that RPL9 gene is overexpressed in colorectal cancer compared to normal colon $(5,13,14)$, which provides the possibility that RPL9 might be involved in tumorigenesis of colorectal cancer.

The aim of the present study was to define the extraribosomal function of RPL9 in colorectal cancer progression. Using RNA interference (RNAi) techniques, we targeted RPL9 gene in colorectal cancer HCT116 and HT29 cells. We found that silencing of RPL9 inhibited colorectal cancer cell growth in vitro as well as in vivo and caused apoptotic cell death. The anti-proliferative effects were induced through a common subset of molecular alterations of 296 mRNA transcripts, including downregulation of Id-1 (inhibitor of DNA binding-1) (15). Western blotting proved that the decrease in Id-1 protein level by RPL9 knockdown was accompanied with the reduction in $\mathrm{I} \kappa \mathrm{B} \alpha$ phosphorylation, which suggests that RPL9 is functionally associated with $\mathrm{Id}-1 / \mathrm{NF}-\kappa \mathrm{B}$ signaling pathway and that the regulation of RPL9 expression may be a potential therapeutic target in colorectal cancer treatment.

\section{Materials and methods}

Cell culture and siRNA transfection. Human colorectal cancer cell lines, HCT116 and HT29, were purchased from the Korean Cell Line Bank (KCLB; Seoul, Republic of Korea). Both HCT116 and HT29 cells were cultured in RPMI-1640 medium (Corning Incorporated, Corning, NY, USA) supplemented with $10 \%$ of fetal bovine serum (FBS; HyClone Laboratories, Logan, UT, USA) and 1\% of penicillin/streptomycin solution (HyClone Laboratories), in a $37^{\circ} \mathrm{C}$ humidified incubator with a mixture of $95 \%$ air and $5 \% \mathrm{CO}_{2}$. The phenotypes of these cell lines have been authenticated by the KCLB. Cells were plated at $30 \%$ density $24 \mathrm{~h}$ before transfection. A total of $15 \mathrm{nM}$ siRNA was mixed with Lipofectamine 2000 (Invitrogen, Carlsbad, CA, USA) in Opti-MEM (Invitrogen) and the medium was replaced $6 \mathrm{~h}$ after transfection. RPL9 siRNA duplexes (si-RPL9) were chemically synthesized by Ambion (Austin, TX, USA; siRNA ID\# s226955). The negative control siRNA (si-NC) that does not target any endogenous transcript was used for control experiments. The sequences of si-NC (Bioneer, Daejeon, South Korea) are as follows: 5'-ACGUGA CACGUUCGGAGAA(UU)-3' (sense) and 5'-UUCUCCGAAC GUGUCACGU-3' (antisense).

Cell proliferation assay. Cell growth was measured using the Cell Counting kit-8 (Dojindo Laboratories, Kumamoto, Japan) according to the manufacturer's instructions. Log phase cells were trypsinized into single cell suspension and HCT116 ( $2 \times 10^{3}$ cells) and HT29 ( $1 \times 10^{3}$ cells) were seeded into 96 -well plates. After $24 \mathrm{~h}$, cells were transfected as described above and after day 1, day 2, day 3 and day 4 of cell culture, the CCK-8 solution was added into each well. After $2 \mathrm{~h}, \mathrm{OD}$ value was measured at a wavelength of $450 \mathrm{~nm}$ using a VersaMax microplate reader (Molecular Devices, Sunnyvale, CA, USA).

Quantitative real-time RT-PCR ( $R T$ - $q P C R)$. The changes in target gene expression were detected using RT-qPCR. Total RNA was isolated using TRIzol (Ambion) and synthesized into cDNA by using first strand cDNA synthesis kit (Takara Bio, Shiga, Japan) according to the manufacturer's instruction. cDNAs were then amplified using corresponding pair of primers (RPL9 forward, 5'-GCACAG TTATCGTGAAGG GC-3' and RPL9 reverse, 5'-TTACCC CACCATTTGTCAA CC-3'; GAPDH forward, 5'-GGGAGCCAAAAGGGTCATCA TCTC-3' and GAPDH reverse, 5'-CCATGCCAGTGAGCTT CCCGTTC-3') synthesized by Macrogen (Seoul, Republic of Korea). The relative quantification of mRNA was measured by LightCycler 96 (Roche, Basel, Switzerland) according to the manufacturer's instructions and quantified using LightCycler 96 software version 1.1, comparing with the $\mathrm{Ct}$ (threshold cycle) values of each target gene. The mRNA levels of GAPDH were used for normalization.

Clonogenic assay. siRNA transfected cells were seeded into three independent wells of 6 -well culture plates $\left(1 \times 10^{3}\right.$ cells/well) and cultured at $37^{\circ} \mathrm{C}$ in $5 \% \mathrm{CO}_{2}$. Cells were maintained without medium change to let the viable cells propagate to sizable colonies for quantification. The colonies were fixed with methanol and then stained with $0.5 \%$ crystal violet for $30 \mathrm{~min}$ at room temperature. The number of colonies formed in each well was counted under the microscope and statistically analyzed.

Cell cycle analysis. Cells were cultured in 60-mm culture dishes and harvested at $72 \mathrm{~h}$ after siRNA transfection. Cells were washed with cold PBS, and then fixed $24 \mathrm{~h}$ with $70 \%$ cold ethanol at $-20^{\circ} \mathrm{C}$. Cells were washed with cold PBS again and incubated in a dark condition with propidium iodide (PI) staining solution containing RNase A (BD Biosciences, San Diego, CA, USA) for $30 \mathrm{~min}$ at room temperature. The cell cycle was measured by FACSVerse flow cytometry (BD Biosciences) according to the manufacturer's instructions and quantified using FlowJo software program.

Detection of apoptosis. Cells were cultured in 60-mm culture dishes and harvested at 48 and $72 \mathrm{~h}$ after siRNA transfection. Apoptotic cells were stained with Annexin V and PI using FITC Annexin V apoptosis detection kit I (BD Biosciences) following the manufacturer's instruction. The cell death was measured by FACSVerse flow cytometry (BD Biosciences) and quantified using FlowJo software program.

RNA sequencing. Total RNA was extracted $48 \mathrm{~h}$ after siRNA transfection using the RNeasy mini kit (Qiagen, Valencia, CA, USA). The quantity of the total RNA was evaluated using RNA electropherograms (Bio-Rad Experion; Bio-Rad Laboratories, 
Hercules, CA, USA); RNA quality was assessed based on the RNA quality indicator (RQI). The total RNA from each sample with a RQI value of 8.0 or higher was used. The resulting mRNA samples were processed for sequencing libraries using the Illumina TruSeq Stranded mRNA sample preparation kit (Illumina, San Diego, CA, USA) according to the manufacturer's protocols. RNA sequencing was performed using the Illumina HiSeq 2500 to generate non-directional, paired-end 100-base-pair reads. Quality-filtered reads were mapped to the human reference genome sequence hg19 (UCSC Genome Bioinformatics, https://genome.ucsc.edu) using TopHat2 (http://ccb.jhu.edu/software/tophat). The relative transcript abundance was estimated by counting the fragments per kilobase of the exon model per million mapped sequence reads (FPKM), and differentially expressed genes were evaluated using the cufflinks package (http://cole-trapnell-lab.github.io/ cufflinks). The significantly overlapping pathways and Gene Ontology categories with differentially expressed genes were analyzed using DAVID (http://david.abcc.ncifcrf.gov) and IPA (ingenuity pathway analysis, www.ingenuity.com).

Western blot analysis. Cells were suspended in RIPA buffer (Thermo Fisher Scientific, Inc., Rockford, IL, USA) containing $0.01 \%$ of a protease and phosphatase inhibitor cocktail (Thermo Fisher Scientific) at $48 \mathrm{~h}$ after siRNA transfection. The amount of protein was quantified by using the Pierce BCA protein assay kit (Thermo Fisher Scientific). Nuclear-cytoplasmic fractionation was conducted using the NE-PER Nuclear and Cytoplasmic Extraction Reagents kit (Thermo Fisher Scientific) according to the manufacturer's protocol. Equal amounts of total proteins were fractionated by SDS-PAGE on a $10 \%$ gel and transferred to PVDF membranes (Roche, Basel, Switzerland). 0.1\% naphthol blue black (NBB) (Sigma-Aldrich, Seoul, Republic of Korea) was used to stain PVDF membrane to confirm equal sample loading. The membrane was blocked with $5 \%$ milk/Tris-buffered saline plus Tween-20 (TBST) and incubated with primary antibodies against human Id-1 (sc-133104), PARP-1 (sc-8007), NF-кB p65 (sc-372), Lamin B1 (sc-30264), $\beta$-actin (sc-47778) (all from Santa Cruz Biotechnology, Santa Cruz, CA, USA), caspase-3 (\#9662; Cell Signaling Technology, Danvers, MA, USA), Phospho-IкB $\alpha$ (Ser32/36) (\#9246; Cell Signaling Technology), GAPDH (NB600-502; Novus Biologicals LLC, Littleton, CO, USA), RPL9 (ab182556; Abcam, Cambridge, MA, USA). HRP goat anti-mouse IgG, HRP goat anti-rabbit IgG and HRP rabbit anti-goat IgG (Santa Cruz Biotechnology) were used as the secondary antibodies. Immunoreactive bands were visualized with an LAS-3000 Imager (Fujifilm Corp., Tokyo, Japan).

Tumorigenic assay in nude mice. Tumor formation was assessed to define the effects of RPL9 silencing on tumorigenicity in vivo. HCT116 cells were transfected with si-NC or si-RPL9 using Lipofectamine 2000. Twenty-four hours after transfection, cells were harvested by trypsinization and then they were washed and resuspended in RPMI mixed with Matrigel (Corning Incorporated). Cells $\left(1 \times 10^{6}\right)$ transfected with si-NC and si-RPL9 were injected subcutaneously into the left and right flank, respectively, of 4-week old male BALB/c nude mice (Orient Bio, Inc., Seongnam, Korea). Tumor formation was monitored at 2-day intervals for 14 days after tumor injection. The tumor size was measured using a vernier caliper and calculated as (width ${ }^{2}$ x length $\mathrm{x} 1 / 2$ ). The mice were then sacrificed and tumor weight of each mouse was evaluated. All the animal experiments were performed in accordance with the guidelines of IACUC (Institutional Animal Care and Use Committee) in Yonsei University Health System with the approval number 2015-0066.

Statistical analysis. All the statistical analysis was performed using Student's t-test, except for in vivo experiment data which was applied with Bootstrap t-test with 10,000 random repetitions. All data are shown as means \pm SEM. P-values $\leq 0.05$, $\leq 0.01$ and $\leq 0.001$ were considered as statistically significant.

\section{Results}

RPL9 knockdown inhibits colorectal cancer cell proliferation and long-term colony formation. To test the effect of RPL9 knockdown on colorectal cancer cell survival, we transfected HCT116 and HT29 cells with si-NC or si-RPL9. After 96 h, we found from microscopic observation that the cell viability of RPL9 knockdown cells had decreased notably (Fig. 1A). In addition, we observed the maximum growth suppression of $\sim 60-80 \%$ in both HCT116 and HT29 cells after 4 days of treatment (Fig. 1B). Concordance with the phenotypic assay result, si-RPL9 effectively silenced target gene expression in both the examined tumor cell lines (Fig. 1C). Consistent with the short-term results, RPL9 knockdown effectively suppressed the long-term colony formation of HCT116 and HT29 cells at day 7 and day 10, respectively, by $\sim 60 \%$ inhibition in both cell lines (Fig. 2). These data indicate that RPL9 is functionally involved in colorectal cell growth.

Silencing of RPL9 induces apoptosis in colorectal cancer cells. To further investigate the role of RPL9 in colorectal cancer growth, the effect of RPL9 silencing on cell cycle progression of HCT116 and HT29 cells were analyzed with flow cytometry (Fig. 3A). It revealed that, when compared to the control treatment, RPL9 knockdown cells showed increased percentage of sub-G1 population and a concomitant decrease in the G1-phase cells in both HCT116 and HT29 cells (Fig. 3B). Since sub-G1 population represents apoptotic cells, we then confirmed the phenomenon by staining CRC cells with the apoptotic marker Annexin V using FACS. Consequently, we affirmed that siRNA knockdown of RPL9 significantly induced the apoptotic cell death at $72 \mathrm{~h}$ after transfection (Fig. 4A). Moreover, the percentages of early-apoptotic cell populations (Q3 region) plus late-apoptotic cell populations (Q2 region) in HCT116 and HT29 cells have increased in both 48 and $72 \mathrm{~h}$ after transfection (Fig. 4B). Early apoptotic cells are considered as cells that have intact plasma membrane which expose phosphatidylserine (PS) on the surface and late apoptotic cells have permeabilized plasma membrane (16). These results suggest that RPL9 knockdown induces apoptosis in colorectal cancer cells.

The function of RPL9 correlates with Id-1/NF- $\kappa B$ signaling. To explore the molecular basis of the growth inhibition caused by RPL9 silencing, we compared the global gene expression profiles of RPL9 knockdown HCT116 and HT29 cells to those 

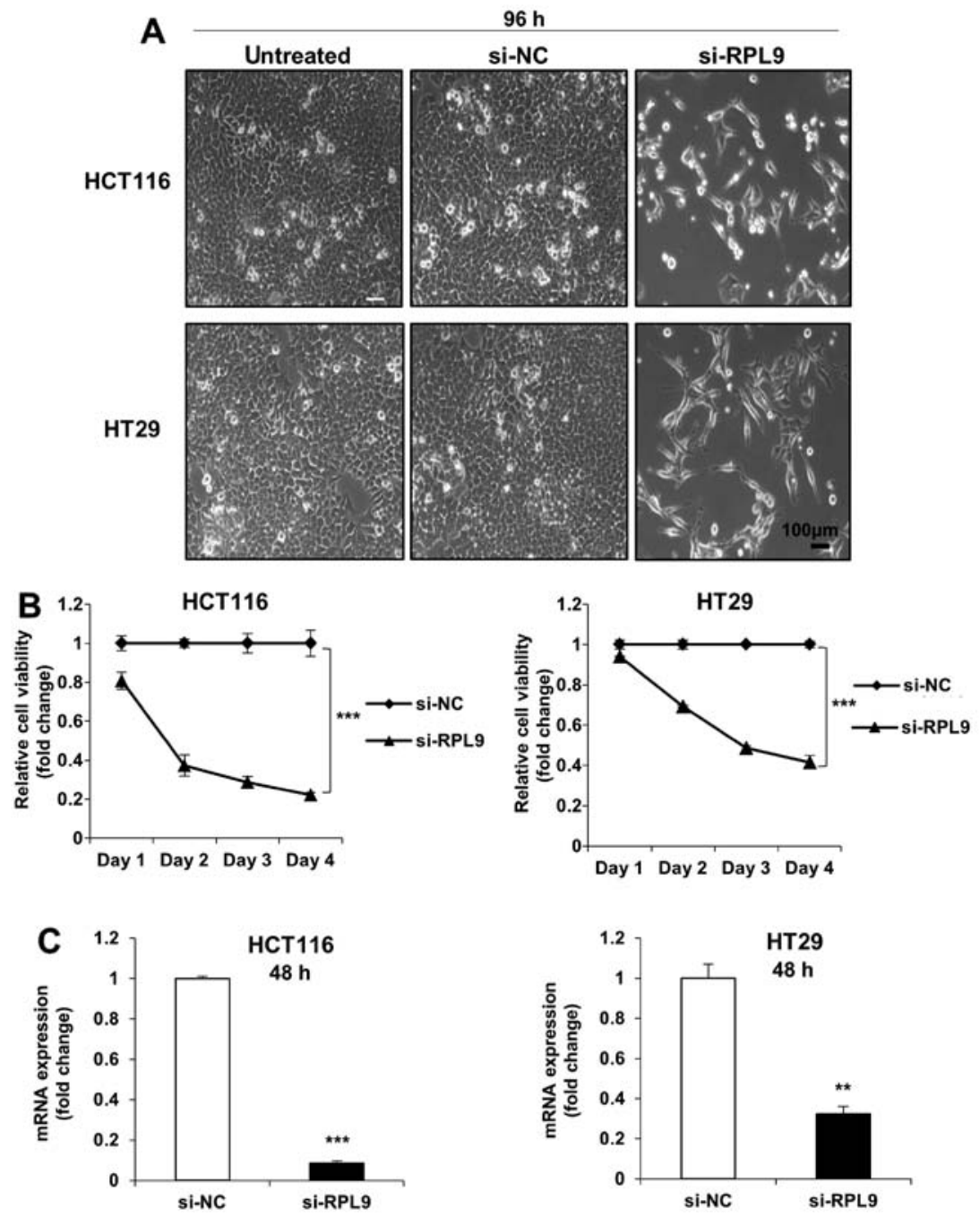

Figure 1. siRNA knockdown of RPL9 inhibits growth of colorectal cancer cells. (A) Representative light microscopy images of HCT116 and HT29 cells $96 \mathrm{~h}$ after transfection. Scale bar, $100 \mu \mathrm{m}$. (B) Detection of cell viability on day 1, day 2, day 3 and day 4 after transfection in HCT116 and HT29 cells. It is expressed as fold changes relative to si-NC. Data represent three independent experiments $\left({ }^{* * *} \mathrm{P}<0.001\right.$ by Student's t-test). (C) Detection of RPL9 mRNA expression in HCT116 and HT29 cells at $48 \mathrm{~h}$ after transfection. The data are shown as means \pm SEM of triplicate experiments $\left({ }^{* * *} \mathrm{P}<0.01 ;{ }^{* * * *} \mathrm{P}<0.001\right.$ by Student's t-test). si-NC, negative control siRNA; si-RPL9, RPL9 specific siRNA.
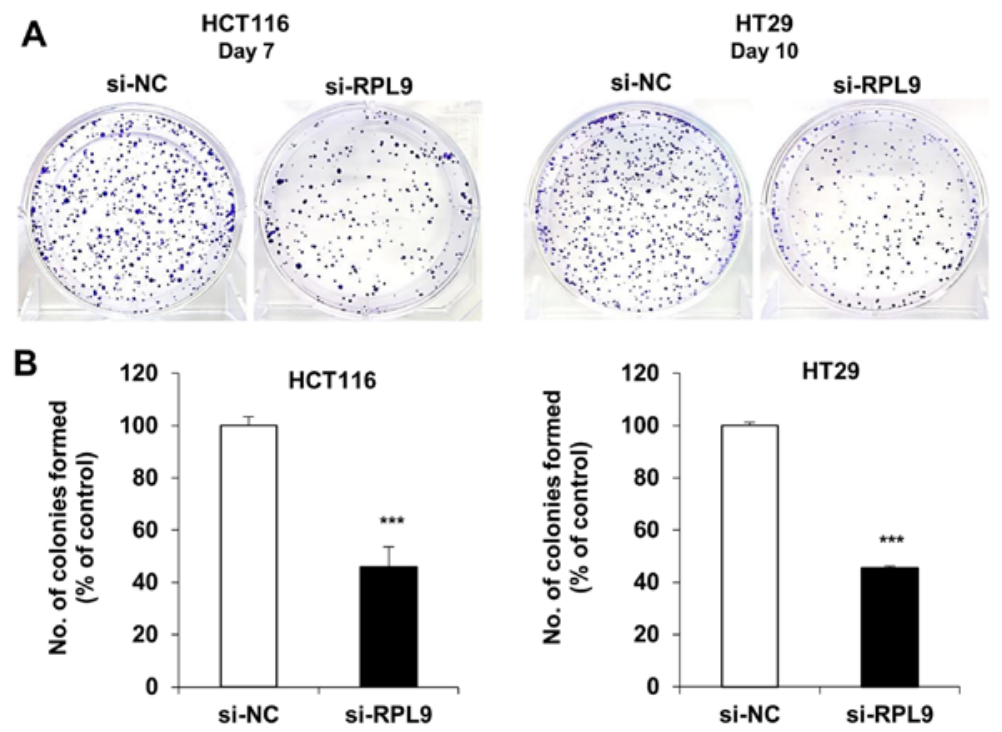

Figure 2. Knockdown of RPL9 reduces long-term colony formation in colorectal cancer cells. (A) Observation of long-term colony formation by clonogenic assay at 7 days and 10 days after transfection in HCT116 and HT29 cells, respectively. (B) The number of colonies was counted and graphed to compare effectively. The data represent three independent experiments $\left({ }^{* * * *} \mathrm{P}<0.001\right.$ by Student's t-test). 
A

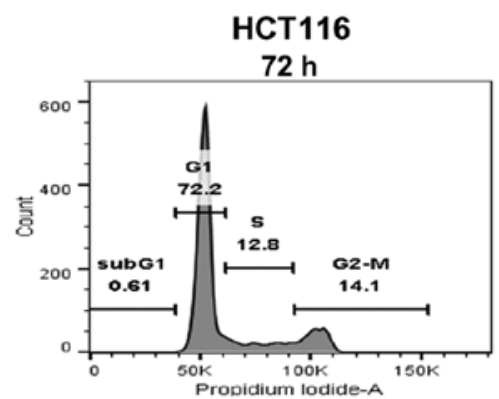

si-RPL9

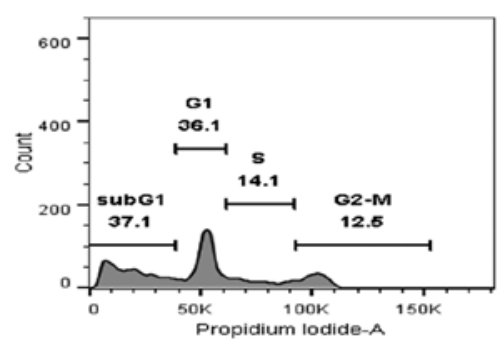

HT29
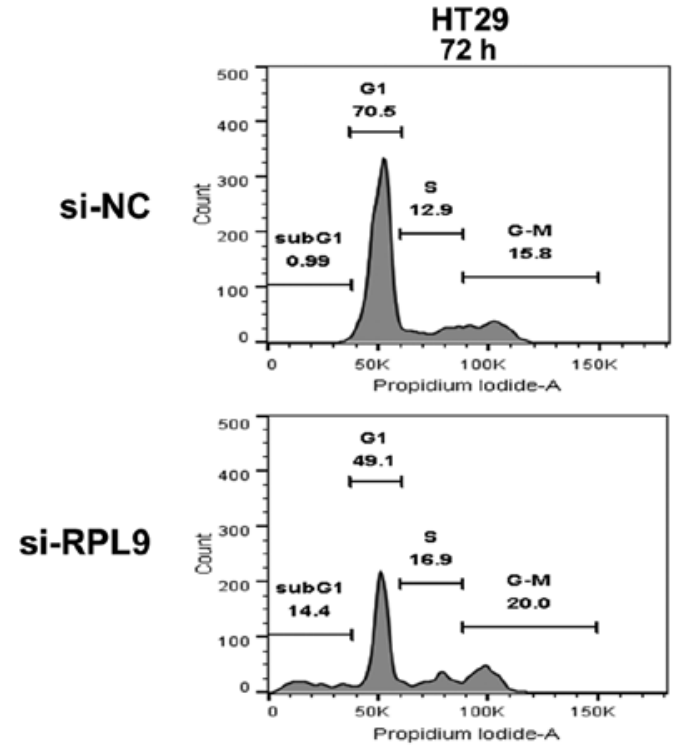
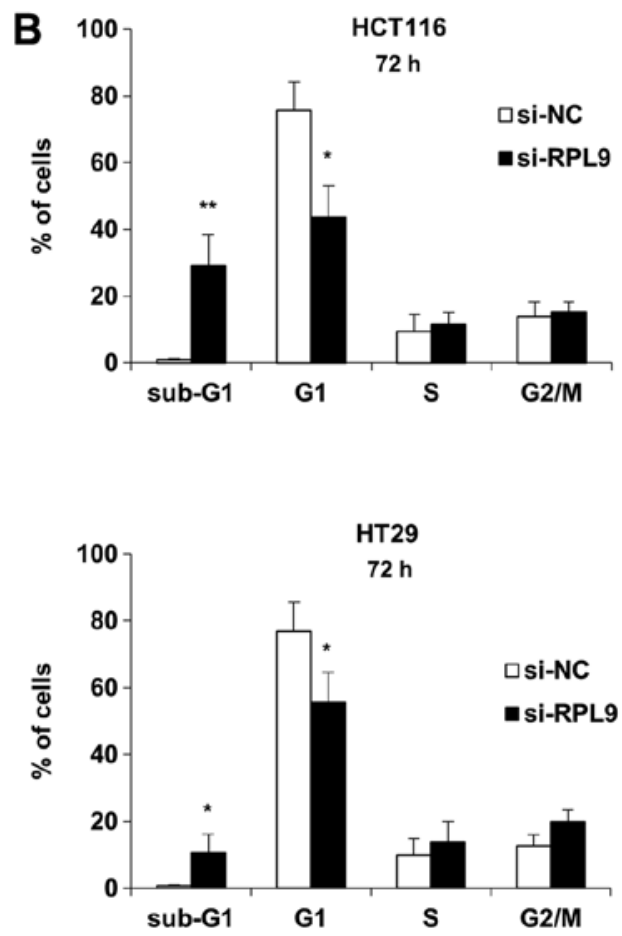

Figure 3. Silencing of RPL9 increases the sub-G1 population in HCT116 and HT29 cells. (A) FACS analysis of cell cycle progression at $72 \mathrm{~h}$ after transfection with si-NC and si-RPL9 is shown in the histogram. (B) For each phase of the cell cycle, percentage of cells is shown in bar graph. The data are shown as means \pm SEM of triplicate experiments $\left({ }^{*} \mathrm{P}<0.05 ;{ }^{* *} \mathrm{P}<0.01\right.$ by Student's t-test).

of control cells transfected with si-NC using next-generation RNA sequencing. The global gene expression analysis, defined as at least a 2.0-fold change, revealed that RPL9-specific knockdown resulted in the upregulation and downregulation of 918 RNA transcripts in HCT116 and 3178 transcripts in HT29 cells (Fig. 5A). Comparing these two gene sets generated a statistically significant overlap of 296 genes (128 upregulated and 168 downregulated genes) which are considered as a common RPL9 knockdown signature (Fig. 5B). Subsequent IPA showed that the 269 mRNA transcripts were functionally enriched in the top five networks (Table I). In particular, as shown in Fig. 5C, we found that the expression level of Id-1, which is involved in carcinogenesis (17) and NF- $\kappa \mathrm{B}$ activation (18), was downregulated in mRNA level by RPL9 silencing. Consistent with our observation, it was previously demonstrated that Id-1 silenced colorectal cancer cells resulted in decreased proliferation and increased apoptotic rate (15). Subsequent western blot analysis confirmed that the protein level of Id-1 was decreased in RPL9 knockdown HCT116 and HT29 cells when compared to the control (Fig. 6A). In addition, a concomitant decrease in pro-caspase 3 , which activate apoptotic signaling, and PARP-1, a nuclear enzyme essential for genomic stability, were observed (Fig. 6A). Meanwhile, IкB $\alpha$ is an inhibitory protein that binds with $\mathrm{NF}-\kappa \mathrm{B}$ in the cytosol making $\mathrm{NF}-\kappa \mathrm{B}$ into an inactivated state. However, when IкB $\alpha$ becomes phosphorylated it dissociates from NF- $\kappa \mathrm{B}$. Then the activated $\mathrm{NF}-\kappa \mathrm{B}$ translocates into the nucleus where it binds to the specific sequences of DNA causing changes in cell function such as promoting cell survival (19). We observed that the protein level of $\mathrm{p}-\mathrm{I} \kappa \mathrm{B} \alpha$ is decreased by RPL9 silencing (Fig. 6A), and NF- $\mathrm{BB}$ protein level also decreased in nucleic fraction of the RPL9 knockdown colorectal cancer cells (Fig. 6B). Since the expression level of housekeeping proteins may vary depending on the experimental condition $(20,21)$, equal sample loading was confirmed by naphthol blue black staining of the western blot membrane (Fig. 6B). These find- 


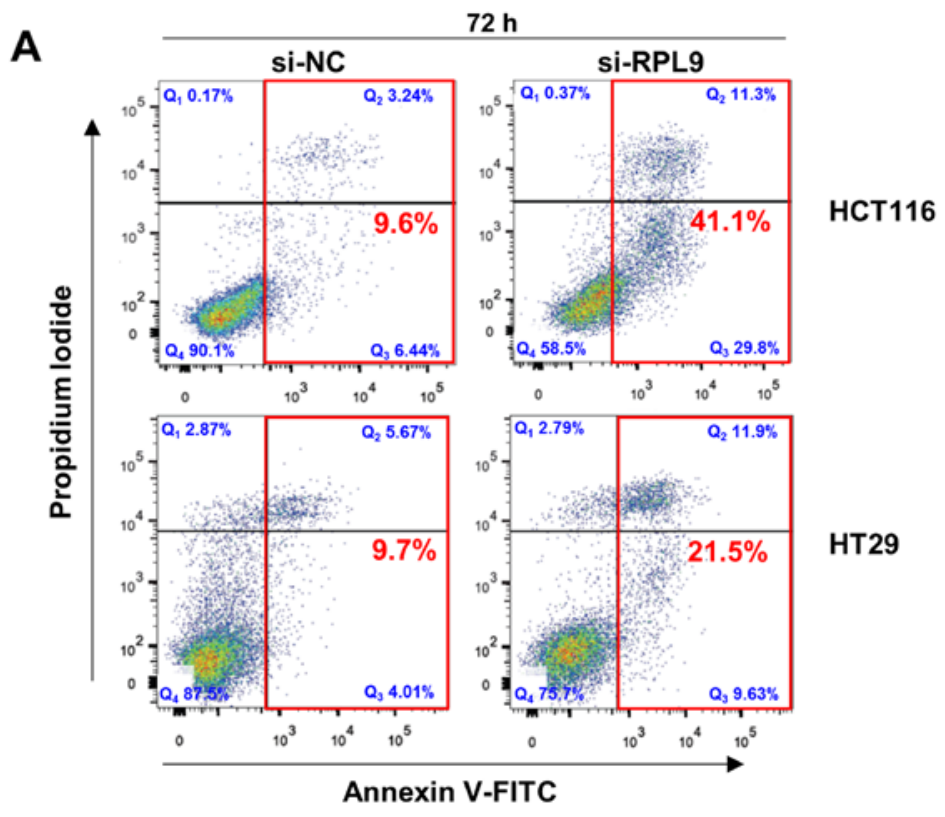

B

HCT116

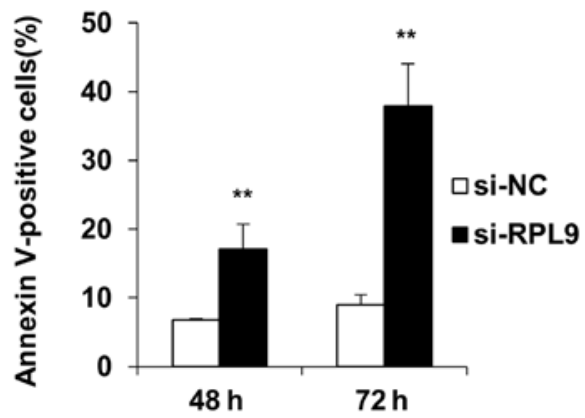

HT29

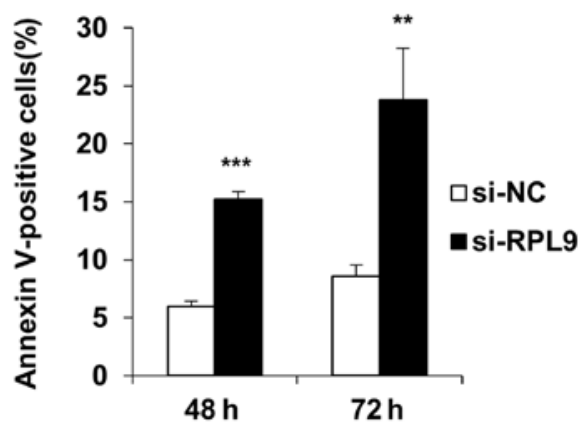

Figure 4. RPL9 silencing induces apoptosis in colorectal cancer cells. (A) Detection of the apoptotic cell population through FACS analysis with the intensity of apoptotic marker Annexin V in HCT116 and HT29 cells at $72 \mathrm{~h}$ after transfection. (B) Number of Annexin V positive cells at 48 and $72 \mathrm{~h}$ after transfection. The data represent three independent experiments $\left({ }^{* *} \mathrm{P}<0.01 ;{ }^{* * *} \mathrm{P}<0.001\right.$ by Student's t-test).

ings indicate that the function of RPL9 is associated with Id-1/ $\mathrm{NF}-\kappa \mathrm{B}$ signaling pathway in colorectal cancer cells.

Silencing of RPL9 delays tumor growth kinetics in vivo. To further validate the effects of RPL9 knockdown on tumorigenesis of colorectal cancer, tumor formation assay was performed in BALB/c nude mice. si-NC and si-RPL9 transfected HCT116 cells were subcutaneously inoculated into the left and right flank, respectively. Tumor sizes were measured in 2-day interval for 14 days. Eight days after the inoculation, tumor sizes between the control and RPL9 knock-down showed definite difference and that the control tumor sizes were larger $(\mathrm{P}<0.05)$ (Fig. 7A). After 14 days, a significant difference $(\mathrm{P}<0.001)$ was apparent in size and weight between the control and RPL9 knock-down (Fig. 7). The results show that silencing of RPL9 significantly suppresses the growth of human CRC xenografts in vivo.

\section{Discussion}

Ribosomal proteins are known as the components of ribosomes which play a role in protein biosynthesis. However, in many types of human cancer cells, some of these ribosomal proteins are ectopically expressed compared to normal cells. For example, the mRNA expression of S8, L12, L23a, L27 and L30 ribosomal proteins was upregulated in human hepatocellular carcinoma (22). RPL34 was overexpressed in non-small cell lung cancer cells (23), and RPS13 was found to promote the growth and cell cycle progression of gastric cancer cells through inhibiting p27 ${ }^{\mathrm{Kip} 1}$ expression (9). Reversely, it was demonstrated that RPS29 induces apoptosis in non-small cell lung cancer cells by downregulating Bcl-2, Bcl-XL and survivin and upregulating p53 and Bax (24). Recent evidence suggests that ribosomal proteins are not only involved in the protein synthesis but also in the development of cancer, and it is likely that these proteins exhibit secondary functions called extraribosomal functions (25).

In colorectal cancer cells, the overexpression of ribosomal protein L9 was found $(5,13,14)$, yet, there is no report on the function of RPL9 in human cancers. In this study we aimed at finding the extraribosomal function of RPL9 in colorectal cancer cells. We used RNAi system to knockdown RPL9 and observed strong inhibition in cell proliferation and colony formation of HCT116 and HT29, which demonstrates that 
A

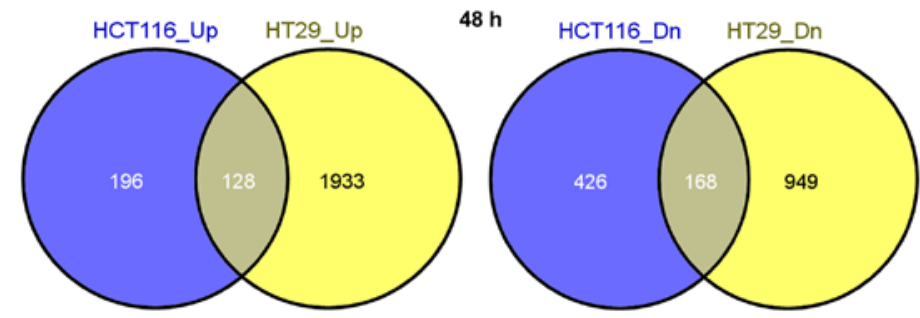

B

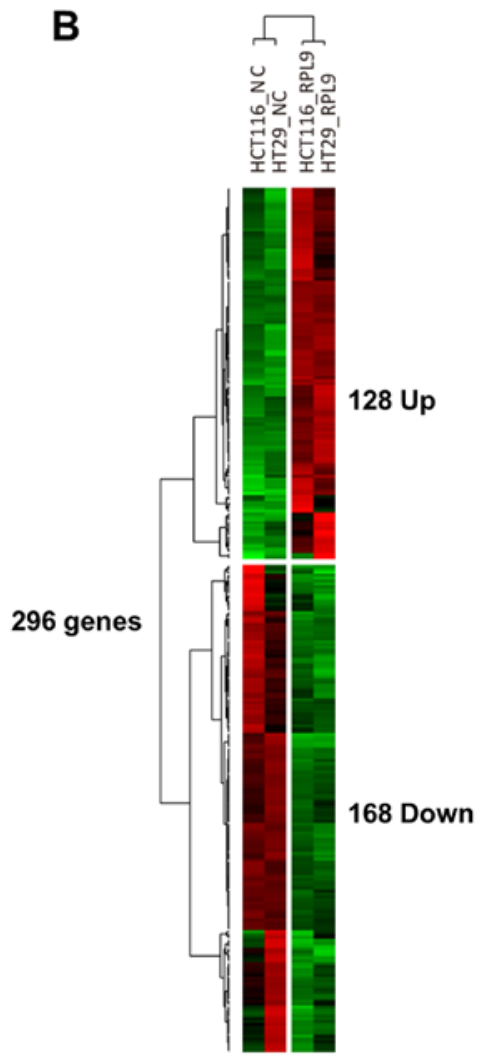

C Network 2

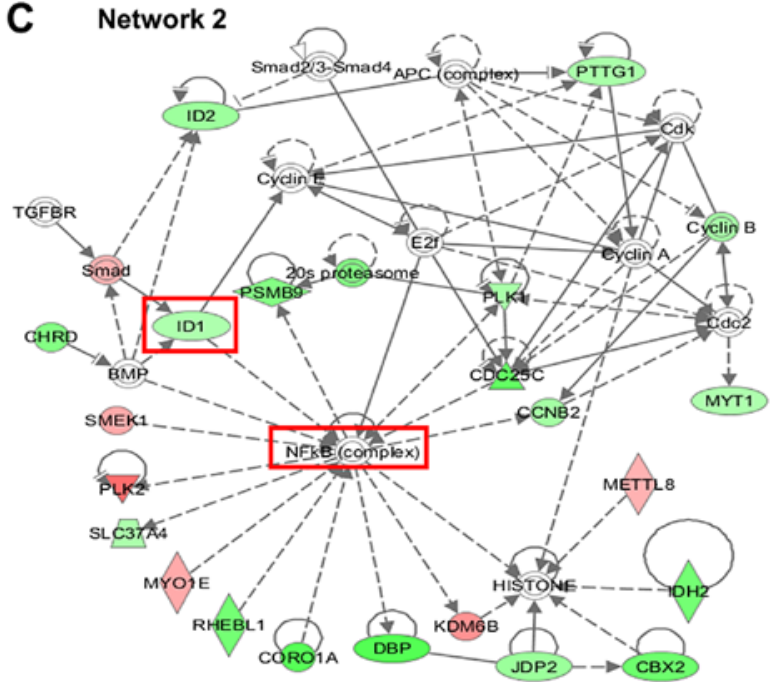

Figure 5. Transcriptomic analysis of RPL9 knockdown gene expression by RNA sequencing. (A) Venn diagrams represent the number of mRNA transcripts upregulated or downregulated at $48 \mathrm{~h}$ after transfection in HCT116 or HT29 cells. (B) A heat map of the 296 commonly deregulated genes in HCT116 and HT29 cells after normalization to the corresponding sham-treated cells [a threshold cut-off of 2-fold change, red (induced) and green (repressed), log2-based scale]. (C) IPA top network 2 associated with Id-1 and NF-кB pathway. Upregulated and downregulated genes by RPL9 silencing are shown in red and green, respectively. The genes shown in gray are associated with the regulated genes.
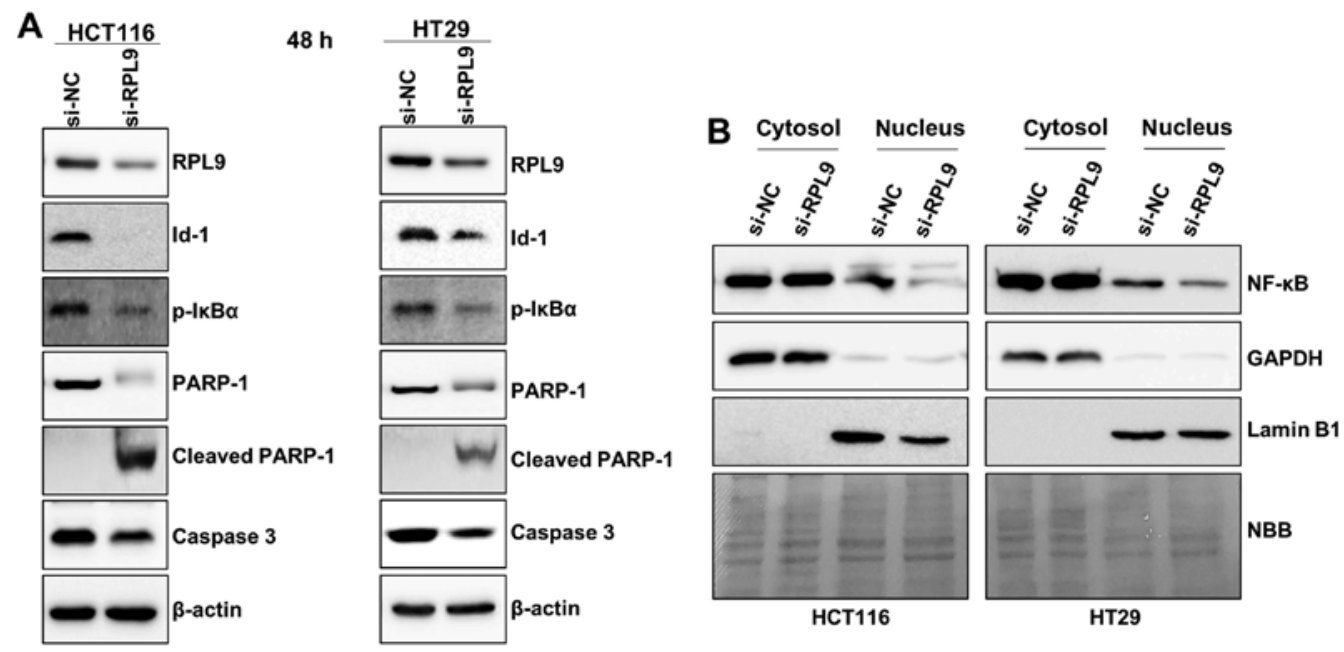

Figure 6. Downregulation of RPL9 causes apoptosis through inactivation of Id-1 and NF-кB. (A) The expression of RPL9, Id-1, p-IкB $\alpha$ (Ser32/36), PARP-1, cleaved PARP-1, caspase-3 in HCT116 and HT29 cells with RPL9 depletion was determined by western blot analysis using the corresponding antibodies. $\beta$-actin was detected as a loading control. (B) Proteins were extracted from both cytosol and nuclei fraction in order to examine localization of NF- $\mathrm{kB}$ p65. Lamin B1 and GAPDH were used as nucleic and cytosolic marker, respectively. Equal sample loading was confirmed by naphthol blue black (NBB) staining of the western blot membrane. 
Table I. Top 5 gene networks from Ingenuity Pathway Analysis.

\begin{tabular}{|c|c|c|c|}
\hline $\begin{array}{l}\text { Network } \\
\text { ID }\end{array}$ & Score & $\begin{array}{c}\text { \# of focus } \\
\text { genes }\end{array}$ & Genes in network and their top functions \\
\hline 1 & 54 & 30 & 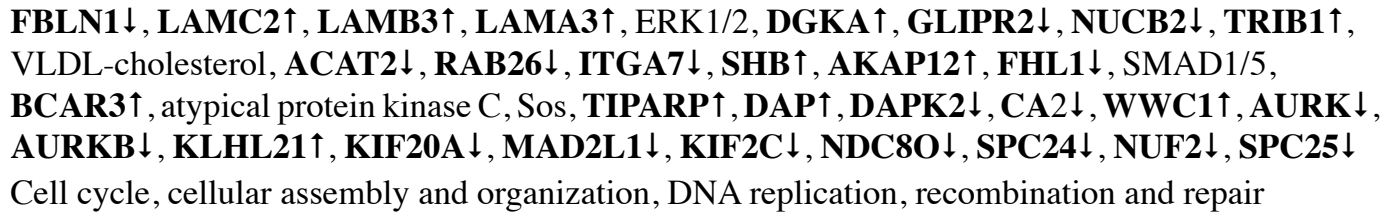 \\
\hline 2 & 33 & 24 & $\begin{array}{l}\text { TGFBR, Smad } \uparrow, \text { ID1 } \downarrow \text {, NFkb (complex), CHRD } \downarrow, \text { BMP, ID2 } \downarrow \text {, cyclin E, SMEK1 } \uparrow, \text { PLK2 } \uparrow, \\
\text { SLC37A4 } \downarrow, \text { MY01E } \uparrow, \text { RHEBL1 } \downarrow, \text { COR01A } \downarrow \text {, DBP } \downarrow \text {, JDP2 } \downarrow, \text { CBX2 } \downarrow \text {, KDM6B } \uparrow, \text { HISTONE, } \\
\text { IDH2 } \downarrow, \text { METTL8 } \uparrow, \text { CCNB2 } \downarrow, \text { CDC25C } \downarrow, \text { PLK1 } \downarrow, \text { 20s proteasome } \downarrow \text {, PSMB9 } \downarrow, \text { E } 2 f, \\
\text { Smad2/3-Smad4, APC (complex), PTTG1 } \downarrow \text {, Cdk, cyclin B } \downarrow \text {, cyclin A, Cdc } 2, \text { MYT1 } \downarrow \\
\text { Cell cycle, DNA replication, recombination and repair, organismal survival }\end{array}$ \\
\hline 3 & 30 & 23 & $\begin{array}{l}\text { IFRD } 1 \uparrow, \text { DES } \downarrow \text {, Pak, ARHGEF } \downarrow \downarrow \text {, Dynamin, ERK, lgG2a, Alpha tubulim, KIF22 } \downarrow \text {, TMEM173 } \downarrow \text {, } \\
\text { STMN } 1 \downarrow \text {, ZFP36L1 } \uparrow, \text { CHAD } \downarrow \text {, LIMA1 } \uparrow, \text { G-Actin } \uparrow, \text { MKNK2 } \uparrow, \text { CaMKII, NFAT (complex), } \\
\text { SDC4 } \uparrow, \text { Nfat (family), KLF6 } \uparrow \text {, Fcer } 1 \text {, calcineurin protein(s), DUSP4 } \uparrow, \text { Cg, MAP2K } 1 / 2, \\
\text { PDGFA } \uparrow, \text { CYR61 } \uparrow, \text { GUSB } \downarrow \text {, HERPUD1 } \downarrow \text {, CTSV } \downarrow \text {, DNASE2 } \downarrow \text {, H2AFZ } \downarrow \text {, HPSE } \downarrow, \text { CENPA } \downarrow \\
\text { Organismal functions, organismal injury and abnormalities, tissue morphology }\end{array}$ \\
\hline 4 & 27 & 22 & $\begin{array}{l}\text { NRGN } \downarrow \text {, thymidine kinase } \downarrow \text {, Rxr, FABP6 } \downarrow \text {, Rar, ITGB3BP } \downarrow \text {, CRABP } \downarrow \downarrow, \text { T3-TR-RXR, } \\
\text { SLC01B3 } \uparrow, \text { Nr1h, PEPCK } \downarrow \text {, PCK } 2 \downarrow, \text { C/ebp, GOT, ANGPTL4 } \uparrow, \text { Growth hormone, PPP1R15A } \uparrow \text {, } \\
\text { STAT5a/b, JINK } 1 / 2 \text {, thyroid hormone receptor, UCP2 } \downarrow, \text { Cbp/p300, PTPase } \downarrow, \text { SLC22A17 } \downarrow \text {, } \\
\text { ACAP1 } \uparrow, \text { Akt, ATF3 } \uparrow, \text { LOC102724788/PRODH } \downarrow, \text { SEMA7A } \uparrow, \text { PLAGL1 } \uparrow \text {, death receptor } \uparrow, \\
\text { TNFRSF10B } \uparrow, \text { TP5313 } \uparrow, \text { TNFRSF1D } \uparrow, \text { HDL cholesterol } \\
\text { Cell death and survival, lipid metabolism, molecular transport }\end{array}$ \\
\hline 5 & 25 & 22 & $\begin{array}{l}\text { MMP17 } \downarrow \text {, Fibrinogen, Ck2, LDL } \downarrow \text {, PSRC } 1 \downarrow \text {, PCSK9 } \downarrow \text {, Pdgf (complex) } \uparrow, \text { Ap } 1, \text { F3 } \uparrow, \text { Creb, } \\
\text { PTPN6 } \downarrow \text {, phosphatase } \downarrow \text {, RNASEH2A } \downarrow \text {, PTPRM } \uparrow, \text { EPHX2 } \downarrow \text {, PPF1A4 } \downarrow \text {, Gsk } 3 \text {, Creb, Ap } 1 \text {, } \\
\text { FSH, DUSP14 } \uparrow \text {, CFLAR } \uparrow, \text { Pkc(s), Pka, Insulin, ATP2A3 } \downarrow, 14-3-3, \text { PP2A, MIDN } \uparrow, \text { Histone } \\
\text { H1 } \downarrow \text {, H1FX } \downarrow \text {, PLC } \downarrow \text {, ADRB, Lh, PTP4A1 } \uparrow, \text { LOC81691 } \downarrow \text {, CRYM } \downarrow \\
\text { Neurological disease, psychological disorders, post-translational modification }\end{array}$ \\
\hline
\end{tabular}

The score is a numerical value used to rank networks according to how relevant they are to the genes in the input dataset (296 genes). The score takes into account the number of genes in network and the size of the network to approximate how relevant this network is to input gene list. Upregulated and downregulated genes (in bold) are indicated with upward and downward arrows, respectively.

RPL9 is important for the growth of colorectal cancer. Also, we found an increased sub-G1 population in cell cycle analysis and likewise, a significant increase of apoptotic cells. Furthermore, in vivo mouse model showed that silencing of RPL9 delayed tumor growth. The global gene expression profiles of RPL9 knockdown HCT116 and HT29 cells to those of control cells by next-generation RNA sequencing showed common knockdown signature of 296 genes. These data suggest that RPL9 is differentially involved in protein synthesis, but does not influence entire cellular protein synthesis in a non-specific manner. Therefore, silencing of RPL9 does not end up downregulating the protein synthesis as a whole but it is rather gene-specific and protein-specific (26).

Of all the genes that were dysregulated by RPL9 knockdown, we focused on finding the particular molecule that might be involved in inducing apoptosis in colorectal cancer cells. Thus, in network 2 of the IPA analysis, we found an oncogenic Id-1 (15) was downregulated. Id-1, a helix-loophelix $(\mathrm{HLH})$ protein, is known to functionally involve in cell growth, senescence and differentiation. Increased Id-1 expression has been found in over 20 types of human cancers including colorectal (27), breast (28), prostate (29) and cervical cancers (30). Many studies suggested that Id-1 plays a key role in cancer progression and associated it as a prognostic marker. According to a study, Id-1 knockdown colorectal cancer cells were decreased in proliferation, migration and invasion and increased in apoptosis rate (15). Therefore, overexpression of Id-1 protects cancer cells against apoptosis, but decreasing the Id-1 expression would lead cancer cells to apoptosis. We found by western blotting that the protein level of Id-1 was decreased in RPL9 knockdown HCT116 and HT29 cells compared to the control. This finding shows that silencing of RPL9 decreased Id-1 protein expression in a mechanism we have not explored and may have induced apoptosis in CRC cells. In addition, Id-1 has been shown to promote cell survival by activating PI3K/Akt/ $N F-\kappa B$ signaling pathway in esophangeal cancer and it has been suggested that it may be one of the upstream regulators 

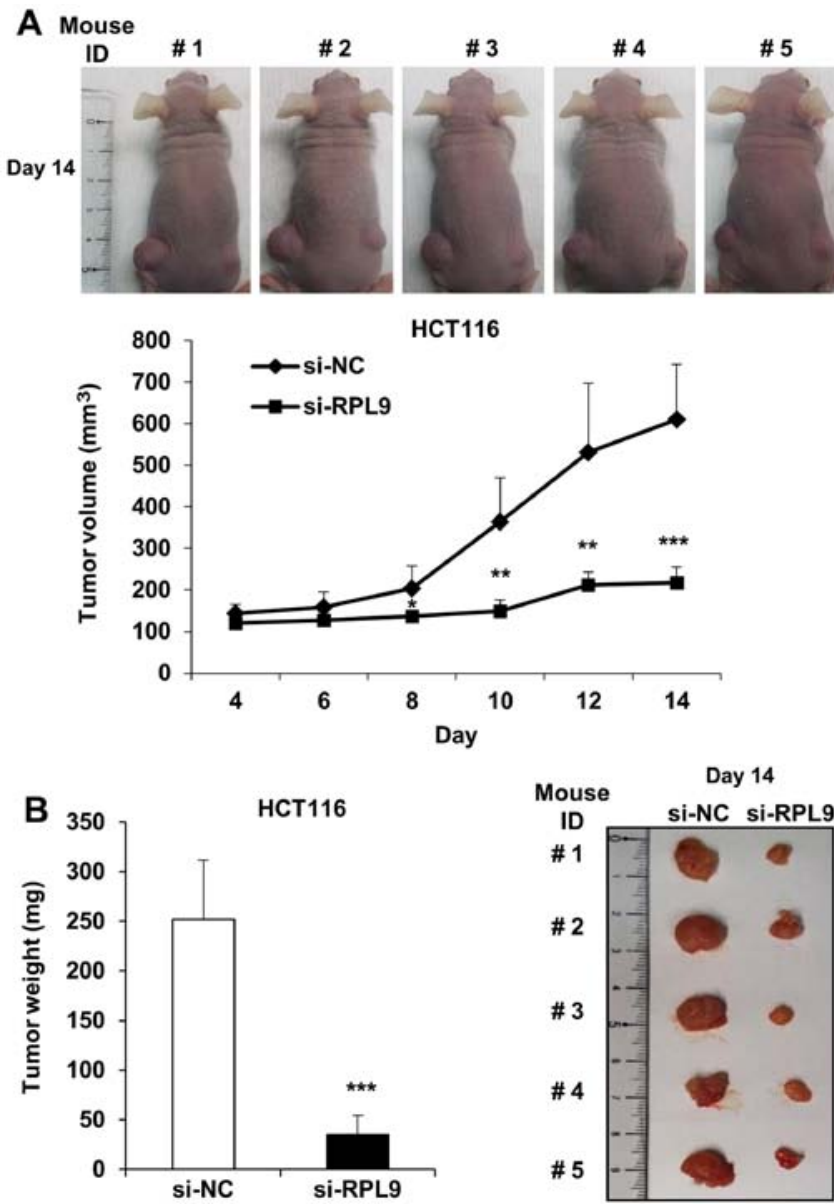

Figure 7. RPL9 silencing suppresses tumor growth in vivo. (A) Observation of tumor sizes between si-NC and si-RPL9 transfected xenografts. Tumor volumes were measured at 2-day intervals for 14 days. The data represent five independent experiments. ${ }^{*} \mathrm{P}<0.05 ;{ }^{* *} \mathrm{P}<0.01 ;{ }^{* * *} \mathrm{P}<0.001$ by Bootstrap t-test. (B) Measurement of the control and RPL9 knockdown tumor weight. The data represent five independent experiments. ${ }^{* * *} \mathrm{P}<0.001$ by Student's t-test.

of $\mathrm{NF}-\kappa \mathrm{B}(18,31)$. NF- $\kappa \mathrm{B}$ is a transcription factor that translocates the nucleus and mediates the transcription of a vast array of proteins involved in cell survival and proliferation, and antiapoptotic factors (32). Hence, we investigated the change in NF- $\kappa$ B activity in RPL9 knockdown HCT116 and HT29 cells and observed that protein level of $\mathrm{p}-\mathrm{I} \kappa \mathrm{B} \alpha$ was decreased and $\mathrm{NF}-\kappa \mathrm{B}$ protein level decreased in the nuclear fraction of RPL9 silenced cells. These results reveal that the downregulation of RPL9 cause inactivation of Id-1 and NF- $\kappa \mathrm{B}$, which leads to apoptosis of colorectal cancer cells.

In Fig. 6B, Lamin B1, a nuclear loading control, shows slight decrease in RPL9 knockdown HCT116 cell. The reason for this may be that Lamin B1 is involved in apoptosis and often cleaved by caspases or becomes degraded $(33,34)$. Depending on the experimental conditions, the expression level of a particular housekeeping gene may change. Hence, there are studies that argue that reversible protein staining dye can be used advantageously over housekeeping protein detection for quality or equal loading control in western blotting $(20,21)$. Therefore, we stained the transferred membrane with naphthol blue black and confirmed equal sample loading of western blotting.
In the present study, we demonstrated for the first time that silencing of RPL9 by RNAi suppresses the proliferation and induces apoptosis in colorectal cancer cells by in vitro and in vivo assays. Also, we found that the downregulation of RPL9 induces the inactivation of Id-1/NF- $\kappa$ B signaling axis. Taken together, our findings suggest that RPL9 may play an important role in promoting the malignant growth of colorectal cancer cells and that targeting of RPL9 might be an attractive option for exploiting a next-line molecular therapy of colorectal cancer.

\section{Acknowledgements}

The present study was financially supported by the grants from the Korean Health Technology R\&D Project, Ministry of Health \& Welfare (A121982) and the Mid-career Researcher Program of the Ministry of Education, Science and Technology (NRF-2014R1A2A1A11054162), Republic of Korea. This study was also supported by the National Research Foundation of Korea (NRF) Grant funded by the Korean Government (MSIP) (NRF-2014R1A5A2010008).

\section{References}

1. Marmot SM, Atinmo T, Byers T, Chen J, Hirohata T, Jackson A, James WPT, Kolonel L, Kumanyika S, et al: Food, Nutrition, Physical Activity, and the Prevention of Cancer: A Global Perspective. WCRF/AICR report: 280-288, 2007.

2. Benson AB III, Venook AP, Bekaii-Saab T, Chan E, Chen YJ, Cooper HS, Engstrom PF, Enzinger PC, Fenton MJ, Fuchs CS, et al; National Comprehensive Cancer Network: Colon cancer, version 3.2014. J Natl Compr Canc Netw 12: 1028-1059, 2014.

3. Sjöblom T, Jones S, Wood LD, Parsons DW, Lin J, Barber TD, Mandelker D, Leary RJ, Ptak J, Silliman N, et al: The consensus coding sequences of human breast and colorectal cancers. Science 314: 268-274, 2006.

4. Taylor DJ, Devkota B, Huang AD, Topf M, Narayanan E, Sali A, Harvey SC and Frank J: Comprehensive molecular structure of the eukaryotic ribosome. Structure 17: 1591-1604, 2009.

5. Lai MD and Xu J: Ribosomal proteins and colorectal cancer. Curr Genomics 8: 43-49, 2007.

6. Fisher EM, Beer-Romero P, Brown LG, Ridley A, McNeil JA, Lawrence JB, Willard HF, Bieber FR and Page DC: Homologous ribosomal protein genes on the human $\mathrm{X}$ and $\mathrm{Y}$ chromosomes: Escape from $\mathrm{X}$ inactivation and possible implications for Turner syndrome. Cell 63: 1205-1218, 1990

7. Draptchinskaia N, Gustavsson P, Andersson B, Pettersson M, Willig TN, Dianzani I, Ball S, Tchernia G, Klar J, Matsson H, et al: The gene encoding ribosomal protein S19 is mutated in Diamond-Blackfan anaemia. Nat Genet 21: 169-175, 1999.

8. Warner JR and McIntosh KB: How common are extraribosomal functions of ribosomal proteins? Mol Cell 34: 3-11, 2009.

9. Guo X, Shi Y, Gou Y, Li J, Han S, Zhang Y, Huo J, Ning X, Sun L, Chen Y, et al: Human ribosomal protein S13 promotes gastric cancer growth through down-regulating $\mathrm{p} 27^{\mathrm{Kip} 1}$. J Cell Mol Med 15: 296-306, 2011.

10. Gou Y, Shi Y, Zhang Y, Nie Y, Wang J, Song J, Jin H, He L, Gao L, Qiao L, et al: Ribosomal protein L6 promotes growth and cell cycle progression through upregulating cyclin $\mathrm{E}$ in gastric cancer cells. Biochem Biophys Res Commun 393: 788-793, 2010.

11. Yang HJ, Youn H, Seong KM, Jin YW, Kim J and Youn B: Phosphorylation of ribosomal protein S3 and antiapoptotic TRAF2 protein mediates radioresistance in non-small cell lung cancer cells. J Biol Chem 288: 2965-2975, 2013.

12. Eid R, Sheibani S, Gharib N, Lapointe JF, Horowitz A, Vali H, Mandato CA and Greenwood MT: Human ribosomal protein L9 is a Bax suppressor that promotes cell survival in yeast. FEMS Yeast Res 14: 495-507, 2014.

13. Zhang XL, Gao F, Li W, Tang WZ and Zhang S: Serial analysis of gene expression in adenocarcinoma samples and normal colonic mucosa in a Chinese population. Genet Mol Res 14: 12903-12911, 2015. 
14. Sim EUH, Bong IPN, Balraj P, Tan SK, Jamal R, Sagap I, Nadeson S, Rose IM and Lim PKM: A preliminary study of differentially expressed genes in Malaysian colorectal carcinoma cases. J Biosci 17: 19-37, 2006.

15. Lai X, Liao J, Lin W, Huang C, Li J, Lin J, Chen Q and Ye Y: Inhibitor of DNA-binding protein 1 knockdown arrests the growth of colorectal cancer cells and suppresses hepatic metastasis in vivo. Oncol Rep 32: 79-88, 2014.

16. Poon IK, Hulett MD and Parish CR: Molecular mechanisms of late apoptotic/necrotic cell clearance. Cell Death Differ 17: 381-397, 2010

17. Meteoglu I, Meydan N and Erkus M: Id-1: Regulator of EGFR and VEGF and potential target for colorectal cancer therapy. J Exp Clin Cancer Res 27: 69, 2008.

18. Ling MT, Wang X, Ouyang XS, Xu K, Tsao SW and Wong YC: Id-1 expression promotes cell survival through activation of NF-kappaB signalling pathway in prostate cancer cells. Oncogene 22: 4498-4508, 2003.

19. Gilmore TD: Introduction to NF-kappaB: Players, pathways, perspectives. Oncogene 25: 6680-6684, 2006.

20. Aldridge GM, Podrebarac DM, Greenough WT and Weiler IJ: The use of total protein stains as loading controls: An alternative to high-abundance single-protein controls in semi-quantitative immunoblotting. J Neurosci Methods 172: 250-254, 2008.

21. Romero-Calvo I, Ocón B, Martínez-Moya P, Suárez MD, Zarzuelo A, Martínez-Augustin O and de Medina FS: Reversible Ponceau staining as a loading control alternative to actin in Western blots. Anal Biochem 401: 318-320, 2010.

22. Kondoh N, Shuda M, Tanaka K, Wakatsuki T, Hada A and Yamamoto M: Enhanced expression of S8, L12, L23a, L27 and L30 ribosomal protein mRNAs in human hepatocellular carcinoma. Anticancer Res 21 (4A): 2429-2433, 2001.

23. Yang S, Cui J, Yang Y, Liu Z, Yan H, Tang C, Wang H, Qin H, Li X, Li J, et al: Over-expressed RPL34 promotes malignant proliferation of non-small cell lung cancer cells. Gene 576: 421-428, 2016.

24. Khanna N, Sen S, Sharma H and Singh N: S29 ribosomal protein induces apoptosis in H520 cells and sensitizes them to chemotherapy. Biochem Biophys Res Commun 304: 26-35, 2003.
25. Naora $\mathrm{H}$ and Naora $\mathrm{H}$ : Involvement of ribosomal proteins in regulating cell growth and apoptosis: Translational modulation or recruitment for extraribosomal activity? Immunol Cell Biol 77: 197-205, 1999.

26. Bee A, Brewer D, Beesley C, Dodson A, Forootan S, Dickinson T, Gerard P, Lane B, Yao S, Cooper CS, et al: siRNA knockdown of ribosomal protein gene RPL19 abrogates the aggressive phenotype of human prostate cancer. PLoS One 6: e22672, 2011.

27. Zhao ZR, Zhang ZY, Zhang H, Jiang L, Wang MW and Sun XF: Overexpression of Id-1 protein is a marker in colorectal cancer progression. Oncol Rep 19: 419-424, 2008.

28. Lin CQ, Singh J, Murata K, Itahana Y, Parrinello S, Liang SH, Gillett CE, Campisi J and Desprez PY: A role for Id-1 in the aggressive phenotype and steroid hormone response of human breast cancer cells. Cancer Res 60: 1332-1340, 2000.

29. Ouyang XS, Wang X, Lee DT, Tsao SW and Wong YC: Over expression of ID-1 in prostate cancer. J Urol 167: 2598-2602, 2002.

30. Schindl M, Oberhuber G, Obermair A, Schoppmann SF, Karner B and Birner P: Overexpression of Id-1 protein is a marker for unfavorable prognosis in early-stage cervical cancer. Cancer Res 61: 5703-5706, 2001

31. Li B, Cheung PY, Wang X, Tsao SW, Ling MT, Wong YC and Cheung AL: Id-1 activation of PI3K/Akt/NFkappaB signaling pathway and its significance in promoting survival of esophageal cancer cells. Carcinogenesis 28: 2313-2320, 2007.

32. Vlahopoulos SA, Cen O, Hengen N, Agan J, Moschovi M, Critselis E, Adamaki M, Bacopoulou F, Copland JA, Boldogh I, et al: Dynamic aberrant NF-кB spurs tumorigenesis: A new model encompassing the microenvironment. Cytokine Growth Factor Rev 26: 389-403, 2015.

33. Broers JL and Ramaekers FC: The role of the nuclear lamina in cancer and apoptosis. Adv Exp Med Biol 773: 27-48, 2014.

34. Goldman RD, Gruenbaum Y, Moir RD, Shumaker DK and Spann TP: Nuclear lamins: Building blocks of nuclear architecture. Genes Dev 16: 533-547, 2002. 